\title{
Four-body continuum-discretized coupled-channels calculations
}

\author{
M. Rodríguez-Gallardo, ${ }^{1,2,3}$ J. M. Arias, ${ }^{2}$ J. Gómez-Camacho, ${ }^{2,4}$ A. M. Moro, ${ }^{2}$ I. J. Thompson, ${ }^{5}$ and J. A. Tostevin ${ }^{6}$ \\ ${ }^{1}$ Instituto de Estructura de la Materia, CSIC, Serrano 123, E-28006 Madrid, Spain \\ ${ }^{2}$ Departamento de Física Atómica, Molecular y Nuclear, Universidad de Sevilla, Apartado 1065, E-41080 Sevilla, Spain \\ ${ }^{3}$ Centro de Física Nuclear, Universidade de Lisboa, Avenida Prof. Gama Pinto 2, P-1649-003 Lisboa, Portugal \\ ${ }^{4}$ Centro Nacional de Aceleradores, Avenida Thomas A. Edison, E-41092 Sevilla, Spain \\ ${ }^{5}$ Lawrence Livermore National Laboratory, Physical Science Directorate, P. O. Box 808, L-414, Livermore, California 94551, USA \\ ${ }^{6}$ Department of Physics, Faculty of Engineering and Physical Sciences, University of Surrey, Guildford, Surrey GU2 7XH, United Kingdom
}

(Received 6 August 2009; published 2 November 2009)

\begin{abstract}
The development of a continuum-bin scheme of discretization for three-body projectiles is necessary for studies of reactions of Borromean nuclei such as ${ }^{6} \mathrm{He}$ within the continuum-discretized coupled-channels approach. Such a procedure, for constructing bin states on selected continuum energy intervals, is formulated and applied for the first time to reactions of a three-body projectile. The continuum representation uses the eigenchannel expansion of the three-body $S$ matrix. The method is applied to the challenging case of the ${ }^{6} \mathrm{He}+{ }^{208} \mathrm{~Pb}$ reaction at $22 \mathrm{MeV}$, where an accurate treatment of both the Coulomb and the nuclear interactions with the target is necessary.
\end{abstract}

DOI: 10.1103/PhysRevC.80.051601

PACS number(s): 21.45.-v, 24.10.Eq, 24.50.+g, 25.60.-t

Rapid experimental developments have made studies of the scattering and reactions of very weakly bound systems possible in many branches of physics. Among these are the Efimov states observed in ultracold cesium trimers [1] and molecular triatomic systems, such as $\mathrm{LiHe}_{2}$ [2], halo nuclei [3], systems undergoing two-proton radioactivity [4], and Bose-Einstein condensates and ultracold dilute gas studies. The need for novel and quantitative theoretical descriptions of the dynamics of such systems, which must include a priori a realistic treatment of their continuum spectra, is common to many problems. Here we discuss continuum effects in the nuclear physics context of reactions of weakly bound halo nuclei $[3,5,6]$. The study of these systems is of special importance in nuclear physics to determine the properties of the nucleon-nucleon interaction in the low-density limit and to investigate the role of pairing off the stability line. Many of these exotic nuclei play a significant role in astrophysical processes. Although the method is general, in this Rapid Communication, we discuss reactions of the Borromean halo nucleus ${ }^{6} \mathrm{He}$, that is, the bound three-body $\left({ }^{4} \mathrm{He}+n+n\right)$ system with (i) no bound excited states and (ii) no bound binary subsystems.

Because of their weak binding, halo nuclei are readily excited to the continuum (broken up) by the differential forces exerted on the constituents through the nuclear and Coulomb interactions with a target. Theoretically, explicit treatment of these breakup channels is difficult, as the states involved are infinite in number and are not square integrable. Hence a robust discretization and truncation scheme, to provide a finite and normalizable basis to represent the continuum, is required. The continuum-discretized coupled-channels (CDCC) method, as formulated for two-body projectiles [7,8], uses bin states to represent the continuum. Here, the continuum spectrum is truncated at a maximum excitation energy $\varepsilon_{\max }$ and the model space, from the breakup threshold to $\varepsilon_{\max }$, is then divided into intervals, where the number and positions of the intervals can depend on the properties (e.g., resonant or nonresonant) of the continuum of the system. For each such interval, or energy bin, a representative square-integrable state is constructed as a linear superposition of the two-body scattering states in the interval. The method has been enormously successful in the description of elastic and breakup observables in reactions involving weakly bound two-body projectiles [9-11] and has recently been extended to include core excitation [12].

Of interest here are reactions of three-body projectiles. To date, published discretized three-body projectile calculations have used a pseudostate basis [13-16], accounting reasonably well for existing elastic scattering data. Based on our own experience [15] with calculations using pseudostate bases, convergence problems are found for reactions where the Coulomb interaction plays an important role. An alternative continuum treatment using the energy-bin technique within the CDCC method had yet to be formulated; the present work provides this theoretical development.

To describe the three-body ground and excited continuum states of the projectile we use a hyperspherical harmonics expansion basis [17]. This involves the use of the one radial and five angular hyperspherical coordinates, $\rho, \alpha, \widehat{x}$, and $\widehat{y}$, obtained from the normalized Jacobi coordinates $\boldsymbol{x}$ and $\boldsymbol{y}$ of the three bodies [15,17]. The quantum number set $\beta$ that defines each three-body channel [15] includes the hypermomentum $K$, the orbital angular momenta $l_{x}$ and $l_{y}$ in coordinates $\boldsymbol{x}$ and $\boldsymbol{y}$, their total $\boldsymbol{l}=\boldsymbol{l}_{x}+\boldsymbol{l}_{y}$, the total spin $S_{x}$ of the particles associated with coordinate $x$, and the intermediate summed angular momentum $\boldsymbol{j}_{a b}=\boldsymbol{l}+\boldsymbol{S}_{x}$. If the spin of the third particle, $I$, is assumed to be fixed, then the total angular momentum is $\boldsymbol{j}=\boldsymbol{j}_{a b}+\boldsymbol{I}$ with projections $\mu$. Note that, for each continuum energy $\varepsilon$ and total angular momentum $j$, there will be as many independent solutions of the three-body scattering problem as there are outgoing channels $\beta$ considered. These solutions can be chosen as the incoming channels $\beta^{\prime}$, but any orthogonal combination of these would be equally valid.

Based on these total angular momentum eigenstates $\mathcal{Y}_{\beta j \mu}(\Omega)[15]$, where $\Omega \equiv(\alpha, \widehat{x}, \widehat{y})$, the bin wave functions 
are defined as

$$
\phi_{n j \mu}^{\mathrm{bin}}(\boldsymbol{x}, \boldsymbol{y})=\sum_{\beta} R_{n \beta j}^{\mathrm{bin}}(\rho) \mathcal{Y}_{\beta j \mu}(\Omega),
$$

where the label $n$ includes reference to the energy interval of the bin $\left[\kappa_{1}, \kappa_{2}\right]$, as well as to the set of quantum numbers $\beta^{\prime}$. The functions $R_{n \beta j}^{\text {bin }}(\rho)$ in Eq. (1) are the associated hyper-radial wave functions,

$$
\begin{aligned}
R_{n \beta j}^{\mathrm{bin}}(\rho) & \equiv R_{\left[\kappa_{1}, \kappa_{2}\right] \beta^{\prime} \beta j}^{\mathrm{bin}}(\rho) \\
& =\frac{2}{\sqrt{\pi N_{\beta^{\prime} j}}} \int_{\kappa_{1}}^{\kappa_{2}} d \kappa e^{-i \delta_{\beta^{\prime} j}(\kappa)} f_{\beta^{\prime} j}(\kappa) R_{\beta \beta^{\prime} j}(\kappa, \rho),
\end{aligned}
$$

where $\kappa=\sqrt{2 m|\varepsilon|} / \hbar$ is the momentum associated with the continuum energy $\varepsilon, R_{\beta \beta^{\prime} j}(\kappa \rho)$ are the continuum hyper-radial wave functions with scattering phase shift $\delta_{\beta^{\prime} j}(\kappa)$, and $f_{\beta^{\prime} j}(\kappa)$ is a weight function with normalization constant $N_{\beta^{\prime} j}$. Note that, for each $j$ and three-body energy bin, we must construct wave functions for all allowed incoming channels $\beta^{\prime}$. Further, as is explicit in Eq. (1), for each $n$ we must also construct $R_{n \beta j}^{\text {bin }}(\rho)$ for all allowed outgoing channels $\beta$.

It follows that the inclusion of a large number of $\beta^{\prime}$ channels is a severe computational challenge and that it is desirable to establish a hierarchy of the continuum states according to their importance to the reaction dynamics. In so doing, we may be able to describe scattering observables using only a selected set of states, their number depending on the reaction under study. To this end, we make use of the eigenstates of the multichannel three-body $S$ matrix [18], or eigenchannels (ECs), as follows. (i) For each $j$ and continuum energy $\varepsilon$, the $S$ matrix in the $\beta$ basis is diagonalized to obtain its ECs, enumerated by $\gamma$, and their corresponding eigenvalues $\exp \left[2 i \delta_{\gamma j}(\kappa)\right]$ and eigenphases $\delta_{\gamma j}(\kappa)$. (ii) The magnitudes of these eigenphases are used to order the ECs. We will show that the ECs with the largest phase shifts are the most strongly coupled in the reaction dynamics, and thus a hierarchy of states can be established by such an ordering. This leads to the possibility of a truncation in the number $n_{\mathrm{ec}}$ of ECs included and testing of the convergence with respect to this number.

Here we apply this methodology to the scattering of ${ }^{6} \mathrm{He}$, treated as a three-body system of an inert $\alpha$-particle core and two valence neutrons. A notable property of ${ }^{6} \mathrm{He}$ is that none of its binary subsystems bind, while the three-body system has a single bound state with a binding energy of $0.973 \mathrm{MeV}$ and total angular momentum $j^{\pi}=0^{+}$. Its low-lying continuum spectrum is dominated by a narrow $j^{\pi}=2^{+}$resonance, $0.825 \mathrm{MeV}$ above threshold. We describe the three-body $\alpha+n+n$ system using the same structure model as in Ref. [15]. The three-body Hamiltonian includes two-body interactions plus an effective three-body potential. For a given value of the maximum hypermomentum used, $K_{\text {max }}$, the parameters of the latter are adjusted to reproduce the ground-state separation energy and matter radius (for $j=0^{+}$) and the resonance energy (for $j=2^{+}$) [15]. Calculations were performed using the codes FACE [19] and STURMXX [20]. The maximum hypermomentum in the $\mathrm{CDCC}$ reaction calculation was $K_{\max }=8$, which provides converged results for the elastic scattering of ${ }^{6} \mathrm{He}$ from a heavy target [15]. The number of
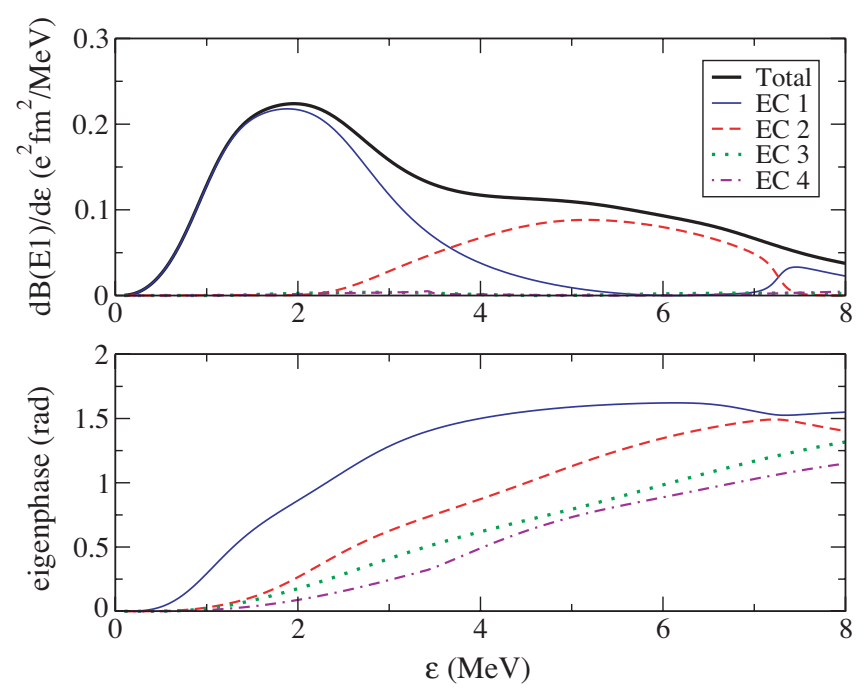

FIG. 1. (Color online) $B(E 1)$ distribution (upper panel) and eigenphase shifts (lower panel) for the first few $j^{\pi}=1^{-}$eigenchannels (ECs) of ${ }^{6} \mathrm{He}$. The thick solid line in the upper panel is the total $B(E 1)$ distribution.

channels $\beta$ was $15\left(0^{+}\right), 26\left(1^{-}\right)$, and $46\left(2^{+}\right)$. The calculated ${ }^{6} \mathrm{He}$ ground state has a binding energy of $0.953 \mathrm{MeV}$ and a single-particle density with a root-mean-square (rms) radius of $2.46 \mathrm{fm}$, assuming an $\alpha$-particle rms radius of $1.47 \mathrm{fm}$.

The EC basis states have properties that are useful for describing collisions. The upper panels in Figs. 1 and 2 show the Coulomb $B(E 1)$ and $B(E 2)$ transition probabilities, respectively, as a function of the ${ }^{6} \mathrm{He}$ excitation energy above threshold. The curves show the total $B(E 1)$ and $B(E 2)$ strengths (thick solid curve) and the contributions from the first four ECs. Because the ${ }^{6} \mathrm{He}$ (g.s.) has $j^{\pi}=0^{+}, B(E 1)$ measures the electric dipole strength to $1^{-}$states, and $B(E 2)$ the quadrupole strength to $2^{+}$states. For each EC, the $1^{-}$and
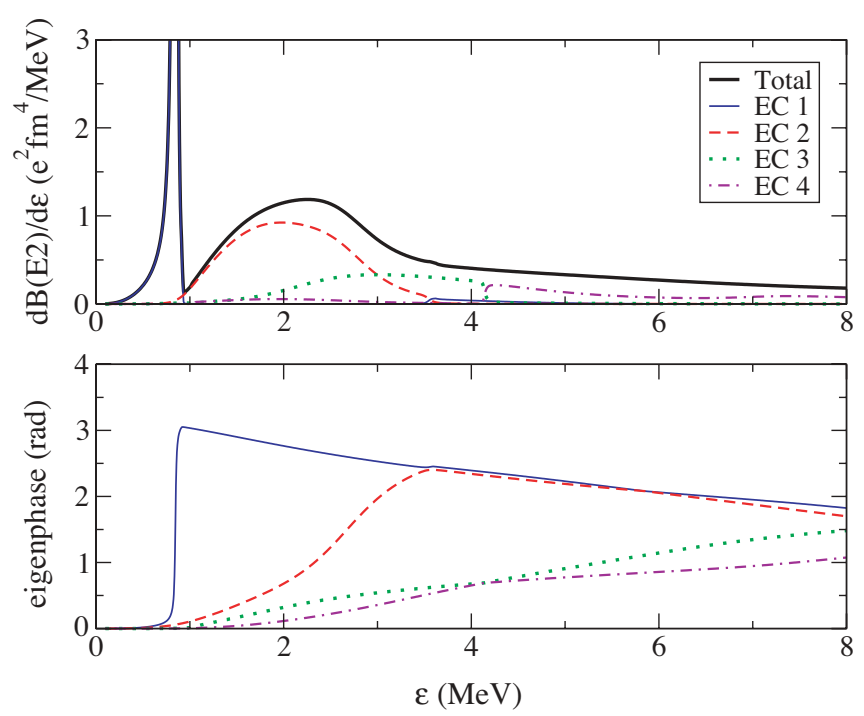

FIG. 2. (Color online) $B(E 2)$ distribution (upper panel) and eigenphase shifts (lower panel) for the first few $j^{\pi}=2^{+}$eigenchannels (ECs) of ${ }^{6} \mathrm{He}$. The thick solid line in the upper panel is the total $B(E 2)$ distribution. 
$2^{+}$eigenphases are shown in the lower panels in Figs. 1 and 2. It has been shown (see, e.g., Ref. [21]) that the position of the peak of the $B(E 1)$ distribution depends on the maximum hypermomentum chosen. In particular, the maximum appears at about $2 \mathrm{MeV}$ for $K_{\max }=10$ and decreases to about $1 \mathrm{MeV}$ for $K_{\max }=20$, for which convergence of this observable is achieved. For $K_{\max }=8$, which is the value used in this work, the maximum is about $2 \mathrm{MeV}$, and hence this observable is not converged. Nevertheless, as we have shown in Ref. [15], the scattering calculations are converged with this value, provided that the parameters of the three-body potential are adjusted such that the ${ }^{6} \mathrm{He}$ system has the same binding energy and rms matter radius for the chosen $K_{\max }$. In principle, the reaction calculations could be done with a higher $K_{\max }$ value, although this would make these calculations computationally very demanding.

It is evident from Figs. 1 and 2 that there is energy localization for the EC contributions to the $E \lambda$ strength. Also evident is that the ECs with eigenphases of the largest magnitude make the maximum contribution to the $B(E \lambda)$ at low excitation energies. This is a very appealing feature for scattering studies where, at moderate collision energies, only continuum states up to a few $\mathrm{MeV}$ play a significant role in the reaction process. So, for example, if excitation energies up to $\approx 8 \mathrm{MeV}$ are strongly populated in a particular reaction, the first three or four ECs should suffice to describe the relevant part of the continuum. Of course, in nuclear collisions near and above the Coulomb barrier, nuclear forces (not included in Figs. 1 and 2) will play an important role. We show here that, with nuclear interactions present, only a few ECs are needed for converged scattering observables.

It is also interesting to identify the dominant quantum numbers of the ECs that make the largest contributions to these observables. For $B(E 1)$ the most important component of ECs 1 and 2 has $l_{n n}=l_{x}=0, S_{x}=0$, or a dineutron configuration. This dominance could explain why previous two-body $(\alpha+2 n)$ model calculations of ${ }^{6} \mathrm{He}$ reactions work to a limited extent, although it is well known that such simple models overestimate continuum coupling effects without parameter adjustments [22,23]. For $B(E 2)$ the most relevant structure is the $2^{+}$resonance that corresponds to EC 1 . In this case we have a combination of three configurations: a dineutron $\left(l_{x}=0, S_{x}=0\right)$, a cigarlike structure $\left(l_{x}=2, S_{x}=0\right)$, and a spin-triplet structure $\left(l_{x}=1, S_{x}=1\right)$.

We now compute the states of Eq. (1) and use these as the basis states for a four-body CDCC calculation using techniques from Ref. [15]. There, a multipole expansion of the coupling potentials was developed for a three-body projectile-plustarget system. The weight function $f_{\gamma j}(\kappa)$ (with $\gamma$ instead of $\beta^{\prime}$ ) used in the superposition of states when constructing the bins, Eq. (2), was taken to be unity for the nonresonant $\left(0^{+}, 1^{-}\right.$, and $\left.2^{+}\right)$continuum and $\sin \left[\delta_{\gamma j}(\kappa)\right]$ for the resonant $\left(2^{+}\right)$continuum. The latter provides an improved description of the resonant character.

Four-body CDCC calculations are carried out for the ${ }^{6} \mathrm{He}+{ }^{208} \mathrm{~Pb}$ reaction at $22 \mathrm{MeV}$, for which experimental data are available in Ref. [24]. This is a demanding example, because (a) long-range Coulomb couplings arising from the heavy target are important and (b) the incident energy is near
TABLE I. Sets of states used in this work for four-body CDCC calculations. $N$ is the total number of states considered (including the ground state). Parentheses are used to specify the two bin schemes adopted, depending on the eigenchannel (set IV) or on the energy (set V).

\begin{tabular}{rcccccr}
\hline \hline Set & $\varepsilon_{\max }(\mathrm{MeV})$ & $n_{\mathrm{ec}}$ & $n_{\text {bin }}\left(0^{+}\right)$ & $n_{\text {bin }}\left(1^{-}\right)$ & $n_{\text {bin }}\left(2^{+}\right)$ & $N$ \\
\hline I & 8 & 4 & 6 & 9 & 6 & 85 \\
II & 8 & 4 & 9 & 12 & 9 & 121 \\
III & 8 & 4 & 12 & 15 & 12 & 157 \\
IV & 8 & $5(1-4,5)$ & $(9,6)$ & $(12,9)$ & $(9,6)$ & 142 \\
V & $9(0-8,8-9)$ & 4 & $(9,1)$ & $(12,1)$ & $(9,1)$ & 133 \\
\hline \hline
\end{tabular}

the Coulomb barrier and both Coulomb and nuclear forces play a significant role.

Implicit in the CDCC calculations is that the actual continuum can be truncated at a maximum excitation energy $\varepsilon_{\max }$, of which the choice is dependent on the specific reaction and projectile energy. The convergence of the model space calculation must then be studied with respect to $\varepsilon_{\max }$ and the number of bins assumed $\left(n_{\text {bin }}\right)$. For our four-body case we must, in addition, study convergence with respect to $n_{\mathrm{ec}}$, the number of ECs included. The present calculations include ${ }^{6} \mathrm{He}$ states with $j^{\pi}=0^{+}, 1^{-}$, and $2^{+}$and projectile-target interaction multipole couplings of the order $Q=0,1,2$. Coulomb and nuclear potentials are included. Nuclear interactions with the target used parametrized optical potentials. For $n+{ }^{208} \mathrm{~Pb}$ and $\alpha+{ }^{208} \mathrm{~Pb}$ the potentials were from Refs. [25] and [26], respectively.

The coupled equations describing the projectile-target motion were solved with the code FRESCO [27], the coupling form factors being read from external files. Partial waves up to $J=150$ were included and the solutions were matched to their asymptotic forms at radius $R_{m}=200 \mathrm{fm}$. To check

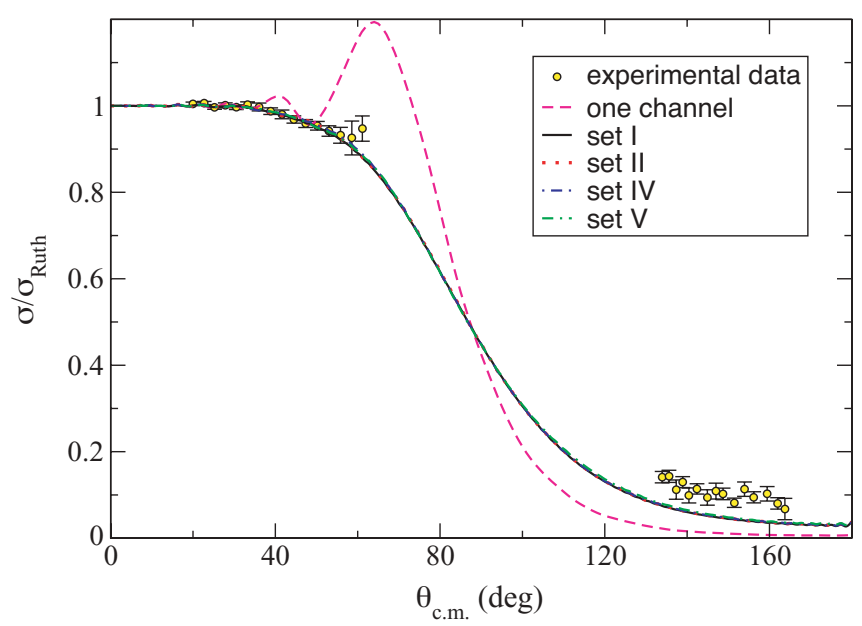

FIG. 3. (Color online) Elastic differential cross section (Rutherford ratio) in the center-of-mass (c.m.) frame for the ${ }^{6} \mathrm{He}+{ }^{208} \mathrm{~Pb}$ reaction at $22 \mathrm{MeV}$. See text for details. Experimental data are taken from Ref. [24]. 


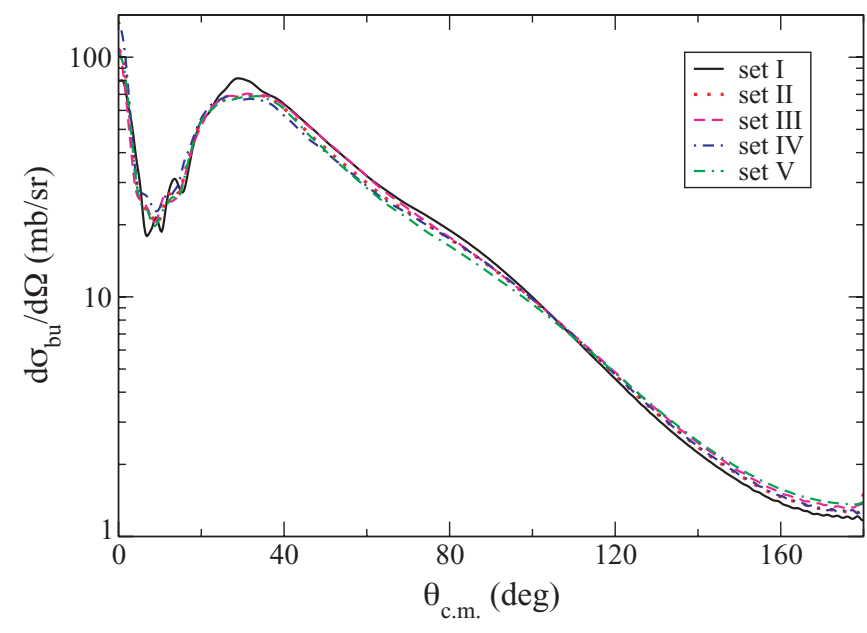

FIG. 4. (Color online) Breakup differential cross-section angular distribution in the center-of-mass (c.m.) frame for the ${ }^{6} \mathrm{He}+{ }^{208} \mathrm{~Pb}$ reaction at $22 \mathrm{MeV}$. See text for details.

the convergence with respect to basis size, for the different observables shown in this work, we use five sets, which are summarized in Table I. Sets I, II, and III have the same maximum energy $\varepsilon_{\max }$ and number of ECs $n_{\mathrm{ec}}$, so they are used to test the convergence with respect to the number of bins considered for each EC and $j^{\pi}$. Set IV is like set II but includes the fifth EC, so it will be used to study the convergence with $n_{\mathrm{ec}}$. Finally, set $\mathrm{V}$ is like set II but includes an additional bin with $\varepsilon=8-9 \mathrm{MeV}$ for all ECs and $j^{\pi}$, providing information on the convergence with respect to $\varepsilon_{\max }$.

First, we analyze the elastic scattering. Figure 3 compares the calculated and experimental (circles) elastic differential cross-section angular distributions (Rutherford ratio). The dashed line is the one-channel calculation, in which continuum states are omitted. The solid (set I), dotted (set II), dot-dashed (set IV), and double-dot-dashed (set V) lines are the full CDCC calculations with different choices of model space. It is remarkable that all these calculations are almost indistinguishable, showing that the elastic angular distribution is converged with set I. Also, this full four-body CDCC calculation, in contrast to the one-channel calculation, describes the data fairly well. Set III is not shown, as convergence with the number of bins is already seen with sets I and II.

Solution of the CDCC coupled equations for the projectiletarget $S$ matrix also provides predictions for breakup observables. First, Fig. 4 shows the breakup differential crosssection angular distribution, summed over the excitation energy. The solid (set I), dotted (set II), dashed (set III), dot-dashed (set IV), and double-dot-dashed (set V) lines are the full CDCC calculations with different choices of model space, as in Fig. 3. For this breakup observable the rate of convergence is slightly slower than for the elastic cross section, although the convergence reached with set II is fairly good.

Second, Fig. 5 shows the breakup cross-section distribution, as a function of the ${ }^{6} \mathrm{He}$ excitation energy above the breakup threshold, for each $j^{\pi}$ and for each EC. This distribution is calculated by dividing the breakup cross section for each bin by its energy width. Here we use set II, which gives a converged result for the breakup angular distribution. As anticipated, in all $j^{\pi}$, the contribution of each EC decreases with decreasing magnitude of its eigenphase. In particular, the contribution of the fourth EC is already small, justifying the use of a truncated calculation and $n_{\mathrm{ec}} \approx 4$. We have also checked that the contribution of the fifth EC (comparing sets II and IV) changes the total breakup cross section by less than $2 \%$. Figure 5 also shows the importance of the dipole and resonant states. These make the largest contribution to the breakup cross section.

Collisions of loosely bound three-body projectiles with a target can be studied in the CDCC framework. The continuumbin discretization procedure has been formulated and used
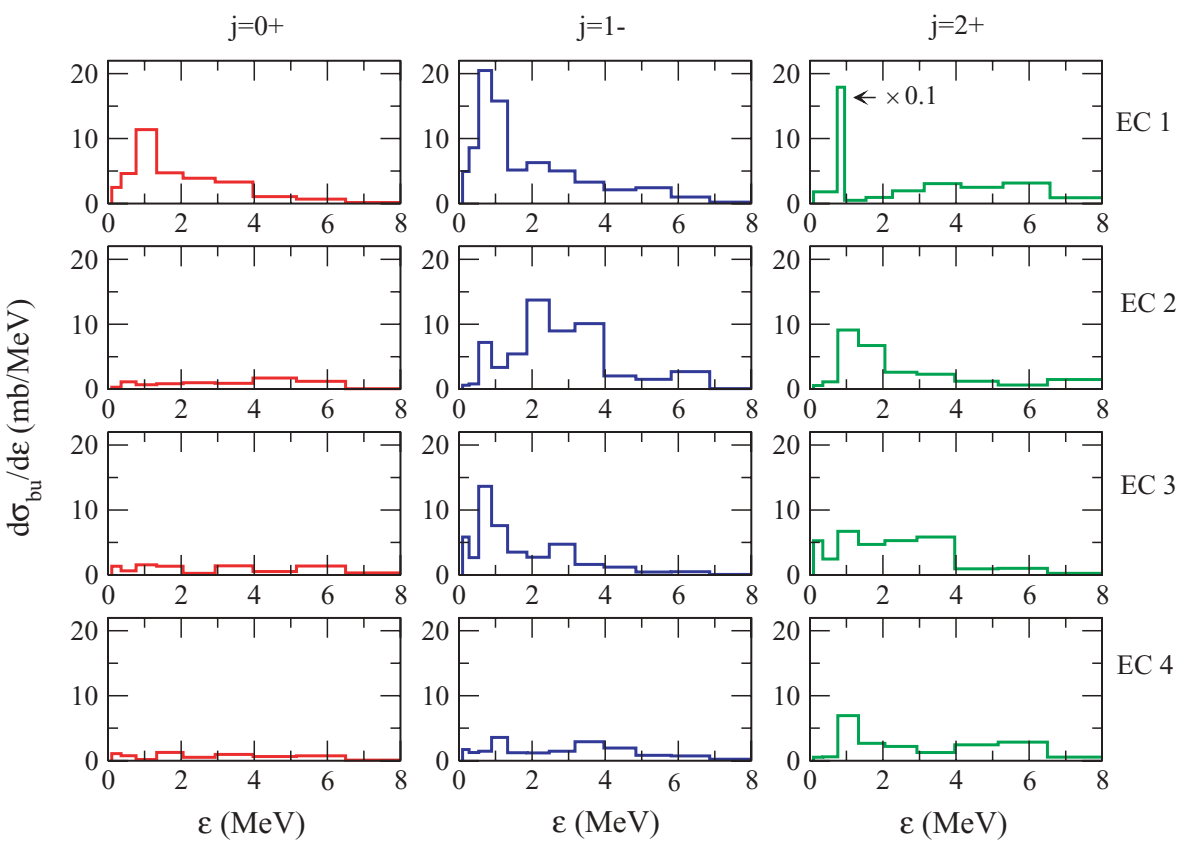

FIG. 5. (Color online) Breakup cross-section distribution for the ${ }^{6} \mathrm{He}+$ ${ }^{208} \mathrm{~Pb}$ reaction at $22 \mathrm{MeV}$ with respect to the ${ }^{6} \mathrm{He}$ excitation energy (over the breakup threshold) for each $j^{\pi}$ and eigenchannel (EC) included in the calculation. Basis set II was used. 
for the first time for a three-body projectile, requiring the superposition of three-body scattering states. For each threebody total angular momentum $j$ and energy $\varepsilon$, scattering states are calculated for ingoing boundary conditions with quantum numbers $\beta$. The multichannel $S$ matrix in this $\beta$ basis, determined from the asymptotics of these states, is diagonalized to determine the ECs, which are ordered according to the magnitude of their associated eigenphases. We have shown that the ECs with the largest eigenphases are most strongly coupled in the collision, suggesting a hierarchy of continuum states and an associated truncation scheme. This EC hierarchy allows practical CDCC calculations that extend the energy-binning procedure to four-body reactions induced by three-body projectiles. The formalism has been applied to the ${ }^{6} \mathrm{He}+{ }^{208} \mathrm{~Pb}$ reaction at $22 \mathrm{MeV}$. The convergence of the results for elastic and breakup cross sections has been presented. Good agreement with existing elastic data for this reaction has been obtained.

We are grateful to F. M. Nunes and R. C. Johnson for useful discussions and suggestions. This work was supported in part by the FCT under Grant No. POCTI/ISFL/2/275 and in part by the DGICYT under Project Nos. FIS 2008-04189 and FPA 2006-13807-C02-01 and the Spanish Consolider-Ingenio 2010 Programme CPAN No. CSD200700042. Part of this work was performed under the auspices of the US Department of Energy at Lawrence Livermore National Laboratory under Contract No. DE-AC5207NA27344. J.A.T. acknowledges the support of the UK Science and Technology Facilities Council under Grant No. EP/D003628. A.M.M. acknowledges a research grant from the Junta de Andalucía.
[1] T. Kraemer et al., Nature (London) 440, 315 (2006).

[2] I. Baccarelli et al., Phys. Chem. Chem. Phys. 2, 4067 (2000).

[3] A. S. Jensen et al., Rev. Mod. Phys. 76, 215 (2004).

[4] I. Muhka et al., Nature (London) 439, 298 (2006).

[5] K. Riisager, Rev. Mod. Phys. 66, 1105 (1994).

[6] P. G. Hansen, A. S. Jensen, and B. Jonson, Annu. Rev. Nucl. Part. Sci. 45, 591 (1995).

[7] M. Yahiro et al., Prog. Theor. Phys. Suppl. 89, 32 (1986).

[8] N. Austern et al., Phys. Rep. 154, 125 (1987).

[9] Y. Sakuragi, M. Yahiro, and M. Kamimura, Prog. Theor. Phys. Suppl. 89, 136 (1986).

[10] F. M. Nunes and I. J. Thompson, Phys. Rev. C 59, 2652 (1999).

[11] J. A. Tostevin, F. M. Nunes, and I. J. Thompson, Phys. Rev. C 63, 024617 (2001).

[12] N. C. Summers, F. M. Nunes, and I. J. Thompson, Phys. Rev. C 74, 014606 (2006).

[13] T. Matsumoto et al., Phys. Rev. C 70, 061601(R) (2004).

[14] T. Matsumoto et al., Phys. Rev. C 73, 051602(R) (2006).

[15] M. Rodríguez-Gallardo et al., Phys. Rev. C 77, 064609 (2008).
[16] T. Egami, T. Matsumoto, K. Ogata, and M. Yahiro, Prog. Theor. Phys. 121, 789 (2009).

[17] B. V. Danilin, T. Rogde, J. S. Vaagen, I. J. Thompson, and M. V. Zhukov, Phys. Rev. C 69, 024609 (2004).

[18] P. Descouvemont, E. Tursunov, and D. Baye, Nucl. Phys. A765, 370 (2006).

[19] I. J. Thompson, F. M. Nunes, and B. V. Danilin, Comput. Phys. Commun. 161, 87 (2004).

[20] I. J. Thompson, Program STURMXX, available from: www.fresco.org.uk/programs/sturmxx/index.html.

[21] B. V. Danilin et al., Nucl. Phys. A632, 383 (1998).

[22] K. Rusek, I. Martel, J. Gómez-Camacho, A. M. Moro, and R. Raabe, Phys. Rev. C 72, 037603 (2005).

[23] A. M. Moro, K. Rusek, J. M. Arias, J. Gómez-Camacho, and M. Rodríguez-Gallardo, Phys. Rev. C 75, 064607 (2007).

[24] A. M. Sánchez-Benítez et al., Nucl. Phys. A803, 30 (2008).

[25] M. L. Roberts et al., Phys. Rev. C 44, 2006 (1991).

[26] A. R. Barnett and J. S. Lilley, Phys. Rev. C 9, 2010 (1974).

[27] I. J. Thompson, Comput. Phys. Rep. 7, 167 (1988). 